\title{
A NEW CHARACTERIZATION OF BARRELLED SPACES
}

\author{
HELMUT PFISTER
}

\begin{abstract}
A locally convex space $E$ is barrelled if and only if $L_{s}(E, F)$ is
\end{abstract} quasicomplete for every Banach space $F$.

Recently A. Wilansky [5] has shown that a locally convex space $E$ is barrelled if for every compact Hausdorff space $X$ and linear map $T: E \rightarrow$ $\mathcal{C}(X)$ with closed graph, $T$ is continuous. His proof runs as follows: For any barrel $B$ in $E$, endow its absolute polar $H:=B^{\circ}$ with the topology induced by the weak topology $\sigma\left(E^{\prime}, E\right)$, form the Banach space $F:=\bigodot^{*}(H)$ of bounded continuous functions on $H$ (which can be identified with $\mathcal{C}(\beta H)$ ), and show that the linear map $T: E \rightarrow F, T(x)(h):=h(x)$ for $x \in E, h \in H$, has closed graph. By hypothesis the continuity of $T$ follows, and so $B$, which is the inverse image of the unit disc in $F$, is a neighborhood of 0 .

Now let $L_{s}(E, F)$ be the space of continuous linear maps of $E$ into $F, s$ indicating the topology of pointwise convergence. We shall show that the continuity of $T$ can be inferred as well, if quasicompleteness of $L_{s}(E, F)$ is supposed. To this end we shall define for every finite set $A \subset E$ and $\varepsilon>0$ a linear map $T_{A, \varepsilon} \in L(E, F)$ such that

$$
\begin{gathered}
\sup \left\{\left\|T_{A, \varepsilon}(x)\right\| ; A \subset E \text { finite, } \varepsilon>0\right\}<\infty \text { for } x \in E, \text { and } \\
\left\|T_{A, \varepsilon}(x)-T(x)\right\|<\varepsilon \text { for } x \in A .
\end{gathered}
$$

Then from (1), (2) and our hypothesis, the continuity of $T$ will be clear.

Let $d$ be the pseudo-metric defined by

$$
d\left(h, h^{\prime}\right):=\sup \left\{\left|h(x)-h^{\prime}(x)\right| ; x \in A\right\} \text { on } H .
$$

Then the uniformity of $(H, d)$ is obviously coarser than the uniformity induced by $\sigma\left(E^{\prime}, E\right)$ on $H$, so $(H, d)$ is precompact. Therefore, there exist $h_{1}, \ldots, h_{n} \in H$ such that $H \subset \cup_{i=1}^{n} K\left(h_{i} ; \varepsilon\right)$ with $K\left(h_{i} ; \varepsilon\right):=\{h \in H$; $\left.d\left(h_{i}, h\right)<\varepsilon\right\}$. From [3, Corollary 5.35 and Problem 5.W] applied to $(H, d)$ and $\left(K\left(h_{i}, \varepsilon\right) ; i=1, \ldots, n\right)$, we get $\varphi_{i} \in \mathcal{C}^{*}(H), i=1, \ldots, n$, with

$$
0 \leqslant \varphi_{i} \leqslant 1, \quad \sum_{i=1}^{n} \varphi_{i}=1, \quad \operatorname{supp} \varphi_{i} \subset K\left(h_{i} ; \varepsilon\right) .
$$

Now let

Received by the editors October 18, 1976.

AMS (MOS) subject classifications (1970). Primary 46A07, 46A20, 46A30. 


$$
T_{A, \varepsilon}(x)(h):=\sum_{i=1}^{n} \varphi_{i}(h) h_{i}(x) \text { for } x \in E, h \in H .
$$

Then obviously $T_{\mathrm{A}, e} \in L(E, F)$, and using (3) one can easily verify that (1) and (2) are satisfied.

Since $L_{s}(E, F)$ is quasicomplete if $E$ is barrelled and $F$ is any quasicomplete locally convex space [1, Chapter III, §3.7, Corollary 2], we obtain the following characterization of barrelledness:

THEOREM. A locally convex space $E$ is barrelled if and only if $L_{s}(E, F)$ is quasicomplete for every Banach space $F$.

A more refined version of this theorem involving cardinals will appear in [4, Satz 2.2]. As a corollary of this, one obtains a characterization of those locally convex spaces $E$ for which Kalton's closed graph theorem [2, Theorem 2.6] is valid by the condition that $L_{s}(E, F)$ is sequentially complete for every separable Banach space $F$.

\section{REFERENCES}

1. N. Bourbaki, Espaces vectoriels topologiques, Chaps. 3-5, Hermann, Paris, 1967.

2. N. J. Kalton, Some forms of the closed graph theorem, Proc. Cambridge Philos. Soc. 70 (1971), 401-408.

3. J. L. Kelley, General topology, Van Nostrand, Princeton, N. J., 1955.

4. H. Pfister, Über das Gewicht und den Überdeckungstyp von uniformen Räumen und einige Formen des Satzes von Banach-Steinhaus, Manuscripta Math. 20 (1977), 51-72.

5. A. Wilansky, On a characterisation of barrelled spaces, Proc. Amer. Math. Soc. 57 (1976), 375.

MATHematisches Institut der Universitït MÜNChen, Theresienstrasse 39, D8 MÜNCHEN 2, Germany 